University of Nebraska - Lincoln

DigitalCommons@University of Nebraska - Lincoln

Faculty Publications, Department of Physics and Astronomy

Research Papers in Physics and Astronomy

3-14-2006

\title{
Comparison of the electronic structure of two polymers with strong dipole ordering
}

\author{
Jie Xiao \\ University of Nebraska-Lincoln, jie.xiao@helmholtz-berlin.de \\ Luis G. Rosa \\ University of Nebraska-Lincoln, luis.rosa13@upr.edu \\ Matt Poulsen \\ University of Nebraska-Lincoln, map@suiter.com \\ Danqin Feng \\ University of Nebraska-Lincoln \\ D. Sahadeva Reddy \\ University of Nebraska-Lincoln \\ See next page for additional authors
}

Follow this and additional works at: https://digitalcommons.unl.edu/physicsfacpub

Part of the Physics Commons

Xiao, Jie; Rosa, Luis G.; Poulsen, Matt; Feng, Danqin; Reddy, D. Sahadeva; Takacs, James M.; Cai, Lei; Zhang, Jiandi; Ducharme, Stephen; and Dowben, Peter A., "Comparison of the electronic structure of two polymers with strong dipole ordering" (2006). Faculty Publications, Department of Physics and Astronomy. 3.

https://digitalcommons.unl.edu/physicsfacpub/3

This Article is brought to you for free and open access by the Research Papers in Physics and Astronomy at DigitalCommons@University of Nebraska - Lincoln. It has been accepted for inclusion in Faculty Publications, Department of Physics and Astronomy by an authorized administrator of DigitalCommons@University of Nebraska Lincoln. 


\section{Authors}

Jie Xiao, Luis G. Rosa, Matt Poulsen, Danqin Feng, D. Sahadeva Reddy, James M. Takacs, Lei Cai, Jiandi Zhang, Stephen Ducharme, and Peter A. Dowben 


\title{
LETTER TO THE EDITOR
}

\section{Comparison of the electronic structure of two polymers with strong dipole ordering}

\author{
Jie Xiao ${ }^{1}$, Luis G Rosa ${ }^{1}$, Matt Poulsen ${ }^{1}$, D-QFeng ${ }^{1}$, \\ D Sahadeva Reddy ${ }^{2}$,James M Takacs ${ }^{2}$, Lei Cai ${ }^{3}$, \\ Jiandi Zhang ${ }^{3}$, Stephen Ducharme ${ }^{1}$ and P A Dowben ${ }^{1,4}$ \\ ${ }^{1}$ Department of Physics and Astronomy and the Center for Materials Research \\ and Analysis, Behlen Laboratory of Physics, University of Nebraska-Lincoln, NE \\ 68588-0111, USA \\ ${ }^{2}$ Department of Chemistry, 316 Hamilton Hall, University of Nebraska-Lincoln, \\ NE 68588-0304, USA \\ ${ }^{3}$ Department of Physics, Florida International University, Miami, FL 33199, USA \\ ${ }^{4}$ Author to whom any correspondence should be addressed: \\ Email: pdowben@unl.edu \\ Received 7 February 2006, in final form 2 March 2006; published 14 March 2006.
}

\begin{abstract}
.
Two different polymers, with large local electric dipoles, are compared: copolymers of polyvinylidene fluoride with trifluoroethylene $[\mathrm{P}(\mathrm{VDF}-\mathrm{TrFE}$, 70\%:30\%)] and polymethylvinylidenecyanide (PMVC). While the different local point group symmetries play a key role, both crystalline polymers exhibit intra-molecular band structure, though the Brillouin zone critical points differ.
\end{abstract}

Polyvinylidene fluoride [PVDF; -(CH2-CF2)n-] copolymers with trifluoroethylene [TrFE;(CHF-CF2)-] can form highly ordered crystalline ferroelectric ultrathin polymer films, as has been demonstrated by x-ray and neutron scattering [1-4], scanning tunnelling microscopy $[1,5-8]$, low energy electron diffraction $[1,8]$ and band mapping $[1,8]$. Although not always evident in scanning tunnelling microscopy, the band structure shows a characteristic super-periodicity dominated by -(CH2-CF2)2-or -(CH2-CF2)-(CHF-CF2)- 'dimer' pairs $[1,8]$ in the ferroelectric phase. The copolymer poly(vinylidene fluoride with trifluoroethylene: 70\%:30\%) [P(VDF-TrFE, 70:30)], in spite of the low overall symmetry, does show all the characteristics of high local symmetry and symmetry selection rules. The effects are quite significant in photoemission [9, 10] and electron energy loss spectroscopy [4, 9-11]. By comparing the copolymer $\mathrm{P}(\mathrm{VDF}-\mathrm{TrFE}, 70: 30)$ to the highly dipole-ordered polymer polymethylvinylidenecyanide (PMVC) we can see the influence of local point group symmetry on the intra-molecular band structure. The results summarized here show that PMVC

Published in J. Phys.: Condens. Matter 18:13 (5 April 2006), pp. L155-L161. doi:10.1088/0953-8984/18/13/L01 http://www.iop.org/EJ/journal/JPhysCM (c) 2006 IOP Publishing Ltd. Used by permission. 
(-(CH(CH3)-C(CN)2)n-) has a much lower local point group symmetry than polyvinylidene fluoride, although not a copolymer system.

The very thin crystalline $\mathrm{P}(\mathrm{VDF}-\mathrm{TrFE}, 70: 30)$ and PMVC films were formed by Langmuir-Blodgett (LB) monolayer deposition from a water subphase. The $\mathrm{P}(\mathrm{VDF}-\mathrm{TrFE}, 70: 30$ ) copolymer with weight-averaged molecular weight $(\mathrm{Mw})=100000$ was dissolved at 0.05 $\mathrm{wt} \%$ in dimethylsulfoxide. The PMVC polymer with weight-averaged molecular weight $(\mathrm{Mw})$ $=2780 \mathrm{was}$ dissolved at $0.07 \mathrm{wt} \%$ in a 90:10 mixture of chloroform and dimethylsulfoxide. For each material, a monolayer was dispersed on the surface of a NIMA model 622C LB trough filled with ultra-pure ( $>18 \mathrm{M} \Omega-\mathrm{cm}$ ) water and compressed to a surface pressure of $5 \mathrm{mN} \mathrm{m}$ 1. The LB films were deposited on substrates of freshly cleaved pyrolytic graphite wafers by the horizontal (Schaefer) variation of the LB technique, repeated five times to make a film of five nominal monolayers (ML). Film preparation methods were described in greater detail in [12]. Previous studies showed that $\mathrm{P}(\mathrm{VDF}-\mathrm{TrFE} 70: 30) \mathrm{LB}$ films produced under these conditions had an average thickness of $1.8 \mathrm{~nm}$ per ML [13]. Prior $\mathrm{x}$-ray diffraction [1, 2], neutron diffraction [4] and scanning tunnelling microscopy $[1,5-8]$ studies of the $\mathrm{P}(\mathrm{VDF}-\mathrm{TrFE} 70: 30) \mathrm{LB}$ films showed that they are highly crystalline and planar, with a crystalline orientation and the polymer chains predominantly parallel to the substrate, but showing little lamellar folding.

Throughout this work, we used nominally $5 \mathrm{ML}$ films of $\mathrm{P}(\mathrm{VDF}-\mathrm{TrFE}, 70: 30)$ and PMVC on graphite for the angle-resolved ultraviolet photoemission spectroscopy (UPS) and inverse photoemission spectroscopy (IPES) experiments. The electron spectroscopy measurements of $\mathrm{P}(\mathrm{VDF}-\mathrm{TrFE}, 70: 30)$ were taken at a sample temperatures of $200 \mathrm{~K}$, well below the $353 \mathrm{~K}$ bulk ferroelectric phase transition of this material $\left(80{ }^{\circ} \mathrm{C}\right)[1,2,7]$, and at room temperature for PMVC. For scanning tunnelling microscopy (STM), the images were recorded at $295 \mathrm{~K}$ from polymer films nominally 2 and $3 \mathrm{ML}$ thick on graphite substrates, for $\mathrm{P}(\mathrm{VDF}-\mathrm{TrFE})$ and PMVC respectively. The STM imaging conditions for P(VDFTrFE) were $V_{\text {tip - bias }}=-0.10 \mathrm{~V}, I=0.21 \mathrm{nA}$. The imaging conditions for PMVC were $V_{\text {tip - bias }}=-0.10 \mathrm{~V}, I=0.2 \mathrm{nA}$.

The UPS and IPES spectra were taken to study the molecular orbital placement of both occupied and unoccupied orbitals of the polymers. In both photoemission and inverse photoemission measurements, the binding energies are referenced with respect to the Fermi edge of gold or tantalum, in intimate contact with the sample surface. The IPES were obtained by using electrons with variable incident energy while measuring the emitted photons at a fixed energy $(9.7 \mathrm{eV})$ using a Geiger-Müller detector $[1,8]$. The instrumental linewidth is $\sim 400 \mathrm{meV}$, as described elsewhere $[1,8]$. The light polarization dependent angle-resolved UPS were found using synchrotron light at $55 \mathrm{eV}$ photon energy, dispersed by a $3 \mathrm{~m}$ toroidal grating monochromator, at the Center for Advanced Microstructures and Devices (CAMD) in Baton Rouge, Louisiana, employing a hemispherical electron energy analyser with an angular acceptance of $\pm 1^{\circ}$, as described in detail elsewhere [14-16]. All angles (both light incidence angles as well as the photoelectron emission angles) reported herein are with respect to the normal to the substrate surface. Because of the highly plane polarized nature of the dispersed synchrotron light through the toroidal grating monochromator, the large light incidence angles result in a vector potential $\mathbf{A}$ more parallel to the surface normal (p-polarized light), while smaller light incidence angles result in the vector potential A residing more in the plane of the surface (s-polarized light) in the geometry of our experiment. The polarization dependence can be related to the photoemission selection rules resulting in changes in the cross-section of symmetry-specific molecular orbitals. The details of selection rule formalism are laid out elsewhere [9, 17-19].

Theoretical calculations of the electronic structure of short segments of $\mathrm{P}(\mathrm{VDF}-\mathrm{TrFE})$ and PMVC were undertaken by NDO-PM3 (neglect of differential overlap, parametric method 3) 


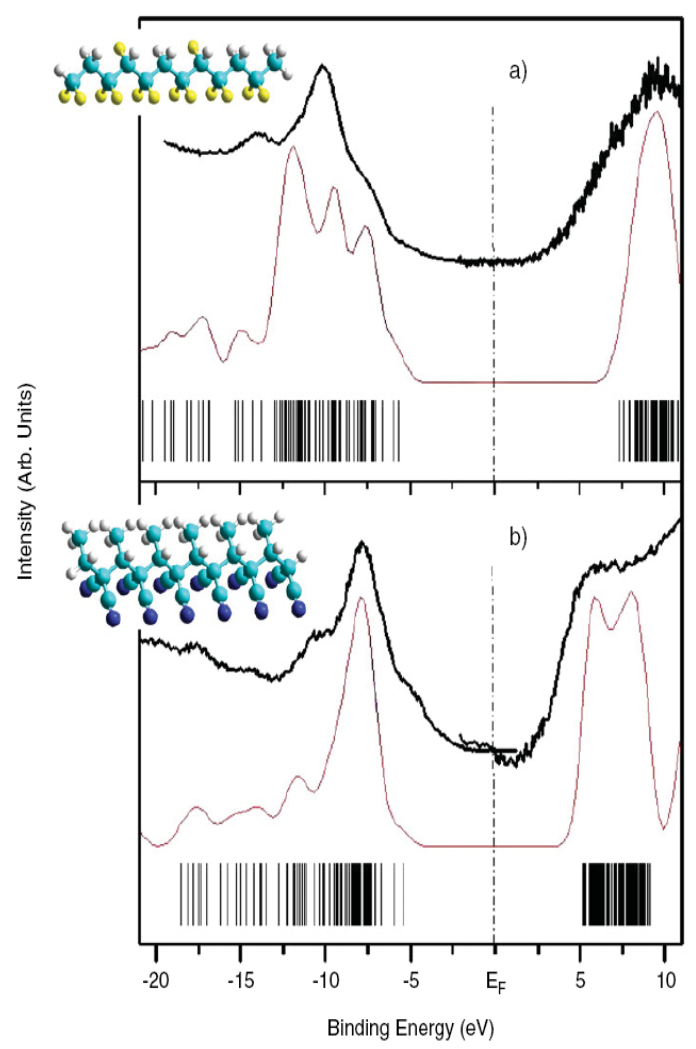

Figure 1. The combined UPS (left) and IPES (right) spectra, along with the calculated molecular orbitals (bottom black lines) and model density of states without any corrections for matrix element or cross-section effects (red line), are shown for $\mathrm{P}(\mathrm{VDF}$ TrFE) copolymer (a) and PMVC (b). The photoemission spectra were taken with the photoelectrons collected normal to the surface, while the inverse photoemission spectrum was taken with the electrons at normal incidence. The insets show schematic representations of chain segments of $\mathrm{P}(\mathrm{VDF}-\mathrm{TrFE})$ in (a) and $\mathrm{PMVC}$ in (b).

with the HyperChem package [20,21]. Geometric optimization of the system was performed by obtaining the lowest unrestricted Hartree-Fock (UHF) energy states: the structures of the short chain segments are shown as insets to figure 1. A calculated density of states (DOS) was obtained by applying equal Gaussian envelopes of $1 \mathrm{eV}$ full width half maximum to each molecular orbital (to account for the solid state broadening in photoemission) and then summing. This calculated density of states, together with a rigid energy shift of $5.3 \mathrm{eV}$ applied to the calculated electronic structure, is in generally good agreement with the combined photoemission and inverse photoemission data from both P(VDF-TrFE, 70:30), taken at 200 $\mathrm{K}$, and PMVC, recorded at $295 \mathrm{~K}$, as seen in figure 1 . The calculations do not account for photoemission and inverse photoemission matrix element effects, so that the comparison with ultraviolet photoemission must be considered only qualitative, but the comparison of theory with experiment, shown in figure 1 , is sufficient to exclude the presence of a significant number of gauche bonds (i.e. such bonds must be below 5\%). The electronic structures of both $\mathrm{P}(\mathrm{VDF}-\mathrm{TrFE}, 70: 30)$, at $200 \mathrm{~K}$, and PMVC, at $295 \mathrm{~K}$, are indicative of systems in the all-trans 


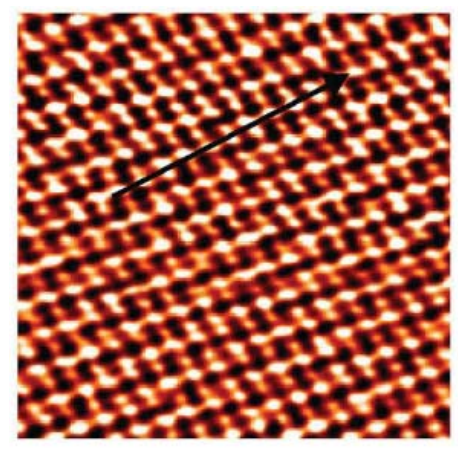

P(VDF-TrFF)

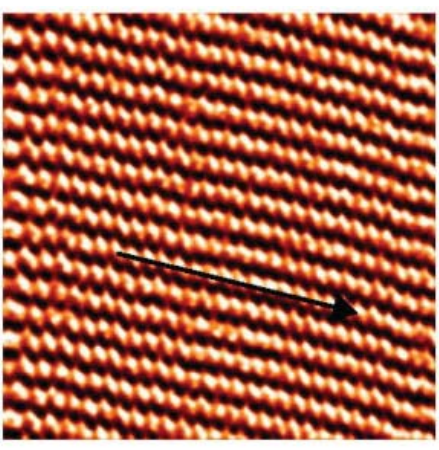

PMVC

Figure 2. The surface structure of crystalline (left) P(VDF-TrFE) and (right) PMVC Langmuir-Blodgett films as ascertained from scanning tunnelling microscopy. The scanning tunnelling microscope images, recorded at $295 \mathrm{~K}$, are of nominally 2 and $3 \mathrm{ML}$ films on graphite substrates, for $\mathrm{P}(\mathrm{VDF}-\mathrm{TrFE})$ and $\mathrm{PMVC}$ respectively. The image sizes are $4.4 \mathrm{~nm} \times 4.4 \mathrm{~nm}$ for $\mathrm{P}(\mathrm{VDF}-\mathrm{TrFE})$ and $4.8 \mathrm{~nm} \times 4.8 \mathrm{~nm}$ for PMVC. Arrows indicate chain direction.

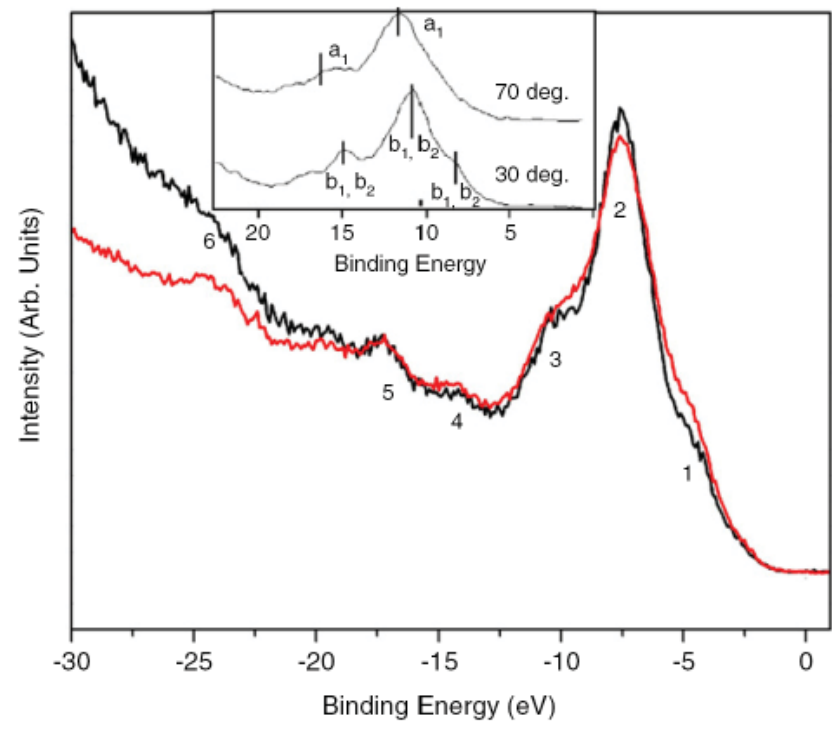

Figure 3. The light polarization photoemission spectra were recorded at a photon energy of $55 \mathrm{eV}$ and $295 \mathrm{~K}$ for PMVC. The $70^{\circ}$ light incidence angle results in $\mathbf{E}$ more along the surface normal (red), while a $45^{\circ}$ light incidence angle reflects $\mathbf{E}$ with components along the surface normal and in the plane of the surface (black). The spectra were taken with the photoelectrons collected at normal emission, to preserve the highest possible point group symmetry. For comparison, the light polarization dependence of $\mathrm{P}(\mathrm{VDF}-\mathrm{TrFE})$ is shown in the inset (adapted from [9]), for $170 \mathrm{~K}$ and light incidence angles of $70^{\circ}$ and $45^{\circ}$, as indicated.

configuration, with the dipoles all aligned. Structural phases that look like 'paraelectric' and 'antiferroelectric' phases are considered very unlikely, though antiparallel packing of chains cannot be excluded on the basis of a comparison of photoemission and inverse photoemission with theory. 
The gap between the highest occupied (HOMO) to lowest unoccupied molecular orbital (LUMO) derived from the combined photoemission and inverse photoemission spectra (figure 1) indicates that $\mathrm{P}(\mathrm{VDF}-\mathrm{TrFE}, 70: 30)$ should be a slightly better dielectric than PMVC, in the absence of structural considerations. This expectation that $\mathrm{P}(\mathrm{VDF}-\mathrm{TrFE})$ is a better insulator is because the HOMO-LUMO gap is slightly larger for $\mathrm{P}(\mathrm{VDF}-\mathrm{TrFE}, 70: 30)$. In addition, the position of the Fermi level within the HOMO-LUMO gap is closer to the lowest unoccupied band edge for PMVC and there is a greater DOS at the lowest unoccupied band edge for PMVC, when compared with that of $\mathrm{P}(\mathrm{VDF}-\mathrm{TrFE})$.

The surface crystallinity of $\mathrm{P}(\mathrm{VDF}-\mathrm{TrFE}, 70: 30)$, grown by the Langmuir-Blodgett technique, has been well established by STM techniques [1,5-8], as already noted. On a cleaved graphite substrate, a $5 \mathrm{ML}$ PMVC film is seen to be similarly crystalline by scanning tunnelling microscopy, as shown in figure 2 . The in-plane spacing between the polymer chains is distributed from 3.5 to $4.3 \AA$ for $\mathrm{P}(\mathrm{VDF}-\mathrm{TrFE}, 70: 30)$, which is somewhat smaller than is generally observed in the bulk crystal structure of $\mathrm{P}(\mathrm{VDF}-\mathrm{TrFE}, 70: 30)$ [2, 22, 23]. Along the chain, as determined from the STM images, the spacing between monomers is about $2.5 \pm 0.1$ $\AA$, in good agreement with the bulk crystal structure of $\mathrm{P}(\mathrm{VDF}-\operatorname{TrFE}, 70: 30)$ [2, 22, 23]. The uniformity of the images tends to exclude antiparallel packing of PMVC and P(VDF-TrFE) chains at the surface.

In spite of the high level of apparent crystalline order, there is very little dependence of the valence band photoemission spectra for PMVC on the incident light polarization (figure 3 ), unlike the considerable light polarization dependent photoemission observed for $\mathrm{P}(\mathrm{VDF}-$ $\operatorname{TrFE}$ ) [9]. For comparison, the significant light polarization dependence of $\mathrm{P}(\mathrm{VDF}-\mathrm{TrFE})$ is shown in the inset (adapted from [9]) in figure 3.

For molecules with high symmetry with respect to the surface normal, the partial cross-section for photoemission varies according to the orientation of the light vector potential $\mathbf{A}$ and the symmetry of the initial state $\psi_{\mathrm{i}}$, assuming that the final state wavefunction $\psi_{\mathrm{f}}$ is fully symmetric, as when the photoelectrons are collected along the surface normal [17-19]. This insensitivity of the photoemission to light polarization effects suggests that PMVC has a much lower local point group symmetry with respect to the surface normal, than $\mathrm{P}(\mathrm{VDF}-\mathrm{TrFE}, 70: 30)$.

PMVC does exhibit significant intra-molecular band structure, particularly in the unoccupied states (figure 4), in spite of the low local point group symmetry. The angle dependent inverse photoemission spectra have been further analysed to determine the dispersion (change in binding energy) as a function of wavevector $\mathbf{k}_{\|}$, parallel to the plane of the surface. The wavevector parallel to the surface of the film follows:

$$
k_{\|}=\sqrt{\frac{2 m}{\hbar} E_{\mathrm{kin}}} \sin (\theta)=0.512 \sqrt{E_{\mathrm{kin}} / e V} \sin (\theta) \AA^{-1}
$$

where the value of $\mathbf{k}_{\|}$(the wavevector parallel with the surface) can be calculated from the kinetic energy $\left(E_{\text {kin }}\right)$ of the incident electron and the incidence angle $\theta$, in the case of inverse photoemission $[8,9,17-19]$.

Angle-resolved inverse photoemission spectra, taken along the molecular chains, show evidence of considerable dispersion. The conduction band dispersion of the lowest unoccupied molecular orbital states, with $\mathbf{k}_{\|}$, along the high symmetry direction is shown in figure 4. The dispersion of the first unoccupied molecular orbital feature along the polymer chain for the $\mathrm{P}(\mathrm{VDF}-\mathrm{TrFE})$ in the ferroelectric phase [2], at $200 \mathrm{~K}$, and room temperature for the PMVC was abstracted from the angle-resolved IPES recorded from multiple samples. The amplitudes of the conduction band dispersion 


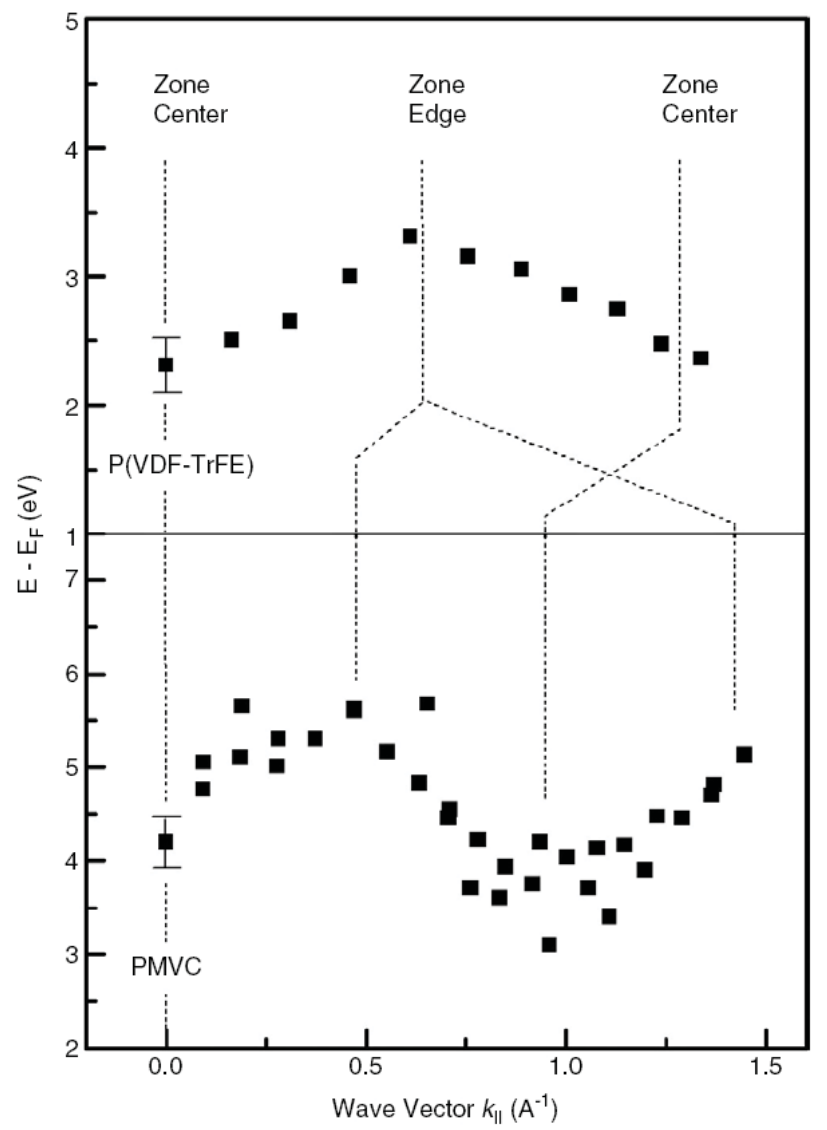

Figure 4. The band dispersion, from angle-resolved inverse photoemission, of the unoccupied feature closest to the Fermi level, along the polymer chains. The dashed lines indicate the different positions of the Brillouin zone centres and Brillouin zone edges between $\mathrm{P}(\mathrm{VDF}-\mathrm{TrFE})$ copolymer and PMVC.

are about 1.5 and $2 \mathrm{eV}$ for $\mathrm{P}(\mathrm{VDF}-\mathrm{TrFE})$ and $\mathrm{PMVC}$, respectively. The real space crystalline periodicity of the conduction band dispersion of $\mathrm{P}(\mathrm{VDF}-\mathrm{TrFE})$ is clearly shorter than for PMVC, judging by the fact that the period in $\mathbf{k}_{\|}$space for $\mathrm{P}(\mathrm{VDF}-\mathrm{TrFE})$ is $1.3 \AA^{-1}$, while, for PMVC the period is $0.9 \AA^{-1}$ in $\mathbf{k}_{\|}$space. As noted previously [1, 8], the length of the Brillouin zone for $\mathrm{P}(\mathrm{VDF}-\mathrm{TrFE})$ of $1.3 \pm 0.4 \AA^{-1}$ is very close to the value $1.255 \AA^{-1}$ which corresponds to $4.8 \pm 0.2 \AA$ or about twice that of the dimer $-\left(\mathrm{CH}_{2}-\mathrm{CF}_{2}\right)_{n}-$ separation of $2.5 \AA$ in real space. For PMVC, the length of the Brillouin zone along the chains is $0.9 \pm 0.6 \AA^{-1}$ in $\mathbf{k}_{\|}$ space, close to $0.84 \AA^{-1}$, which corresponds to three times the dimer distance in real space or $7 \pm 0.5 \AA$. The reason why the size of the unit cell of PMVC in real space is $7 \pm 0.5 \AA$ may be a natural arrangement of the methyl groups or, as has been suggested for $\mathrm{P}(\mathrm{VDF}-\mathrm{TrFE})[1,5$, $6,8]$, due to some subtle local arrangements of dipole orientation.

It is very clear from scanning tunnelling microscopy (figure 3) and band structure (figure 4) that PMVC, like $\mathrm{P}(\mathrm{VDF}-\mathrm{TrFE})$, can be highly ordered when the films are prepared by the Langmuir-Blodgett technique. Indeed, the absence of significant light polarization effects in photoemission, combined with the high level of crystallinity, tends to indicate that an overall low symmetry matters less than local symmetry and dipole ordering. 
This work was supported by the National Science Foundation through grant CHE-0415421 and the NSF ' QSPINS' MRSEC (DMR 0213808). The Center for Advanced Microstructures and Devices (CAMD) is funded by the Louisiana Board of Regents.

\section{References}

[1] Choi J, Borca C N, Dowben P A, Bune A, Poulsen M, Pebley S, Adenwalla S, Ducharme S, Robertson L, Fridkin V M, Palto S P, Petukhova N N and Yudin S G 2000 Phys. Rev. B 61 5760-70

[2] Blinov L M, Fridkin V M, Palto S P, Bune A V, Dowben P A and Ducharme S 2000 Usp. Fiz. Nauk 170 247-62

Blinov L M, Fridkin V M, Palto S P, Bune A V, Dowben P A and Ducharme S 2000 Phys. Usp. 43 243-57 (Engl. Transl.)

[3] Jacobson P A, Rosa L G, Othon C M, Kraemer K, Sorokin A V, Ducharme S and Dowben P A 2004 Appl. Phys. Lett. 84 88-90

[4] Borca C N, Adenwalla S, Choi J, Sprunger P T, Ducharme S, Robertson L, Palto S P, Liu J, Poulsen M, Fridkin V M, You H and Dowben P A 1999 Phys. Rev. Lett. 83 4562-5

[5] Qu H, Yao W, Garcia T, Zhang J, Sorokin A V, Ducharme S, Dowben P A and Fridkin V M 2003 Appl. Phys. Lett. 824322

[6] Cai L, Qu H, Lu C, Ducharme S, Dowben P A and Zhang J 2004 Phys. Rev. B 70155411

[7] Bune A V, Fridkin V M, Ducharme S, Blinov L M, Palto S P, Sorokin A V, Yudin S and Zlatkin A 1998 Nature 391874

[8] Choi J, Dowben P A, Ducharme S, Fridkin V M, Palto S P, Petukhova N and Yudin S G 1998 Phys. Lett. A 249505

[9] Choi J, Tang S-J, Sprunger P T, Dowben P A, Fridkin V M, Sorokin A V, Palto S P, Petukhova N and Yudin S G 2000 J. Phys.: Condens. Matter 12 4735-45

[10] Rosa L G, Losovyj Ya B, Choi J and Dowben P A 2005 J. Phys. Chem. B 109 7817-20

[11] Choi J, Morikawa E, Ducharme S and Dowben P A 2005 Mater. Lett. 59 3599-603

[12] Ducharme S, Palto S P and Fridkin V M 2002 Ferroelectric polymer Langmuir-Blodgett films Ferroelectric and Dielectric Thin Films ed H S Nalwa (San Diego, CA: Academic)

[13] Bai M et al 2004 J. Appl. Phys. 95 3372-76

[14] McIlroy D N, Waldfried C, McAvoy T, Choi J, Dowben P A and Heskett D 1997 Chem. Phys. Lett. $264168-73$

[15] Choi J, Chipara M, Xu B, Yang C S, Doudin B and Dowben P A 2001 Chem. Phys. Lett. 343193

[16] Dowben P A, LaGraffe D and Onellion M 1989 J. Phys.: Condens. Matter 16571

[17] Dowben P A, Choi J, Morikawa E and Xu B 2002 The band structure and orientation of molecular adsorbates on surfaces by angle-resolved electron spectroscopies Handbook of Thin Films vol 2 (San Diego, CA: Academic) chapter 2 pp 61-114

[18] Steinrück H-P 1996 J. Phys.: Condens. Matter 8 6465-509

[19] Plummer E W and Eberhardt W 1982 Adv. Chem. Phys. 49533

[20] Stewart J J P 1989 J. Comput. Chem. 10209

[21] Stewart J J P 1989 J. Comput. Chem. 10221

[22] Furukawa T 1989 Phase Transit. 18143

[23] Legrand J F 1989 Ferroelectrics 91303 